\title{
Friction of rough surfaces on ice: experiments and modeling
}

\author{
Alberto Spagni ${ }^{1}$, Alice Berardo², Diego Marchetto1,3 *, Enrico Gualtieri ${ }^{3}$, Nicola M. Pugno ${ }^{2,4,5}$, \\ Sergio Valeri1 13,6 \\ ${ }^{1}$ Dipartimento di Scienze Fisiche, Informatiche e Matematiche -Università di Modena e Reggio Emilia, \\ Via Campi 213/A - 41125 Modena, Italy \\ ${ }^{2}$ Laboratory of Bio-inspired \& Graphene Nanomechanics, Department of Civil, Environmental and Mechanical Engineering, \\ University of Trento, Via Mesiano 77, 38123 Trento, Italy \\ ${ }^{3}$ CNR-Istituto di Nanoscienze, Centro S3, Via Campi 213/A -41125 Modena, Italy \\ ${ }^{4}$ Center for Materials and Microsystems, Fondazione Bruno Kessler, Via Sommarive 18, 38123 Povo (TN), Italy \\ ${ }^{5}$ School of Engineering and Materials Science, Queen Mary University of London, Mile End Road, London E1 4NS, U.K. \\ ${ }^{6}$ Centro Interdipartimentale per la Ricerca Applicata e i Servizi nel settore della Meccanica Avanzata \\ e della Motoristica - Università di Modena e Reggio Emilia, Via Vivarelli 2 - 41125 Modena (Italy). \\ * Corresponding author. \\ E-mail address: diego.marchetto@unimore.it (D. Marchetto).
}

\begin{abstract}
Over a century of scientific research on the sliding friction of ice has not been enough to develop an exhaustive explanation for the tribological behavior of frozen water. It has been recognized that ice shows different friction regimes, but a detailed description of all the different phenomena and processes occurring at the interface, including the effect of surface roughness of both the ice and the antagonist material is still missing.

In this work the effect of surface morphology on the friction of steel/ice interfaces is studied. Different degrees of random roughness on steel surfaces are introduced and the friction coefficient is measured over a wide range of temperature and sliding velocity. Correlation between the surface roughness and the lubrication regime and friction coefficient is discussed. A theoretical model is developed in order to explain this correlation, and to control the tribological behavior of the system by a proper selection of surface roughness parameters.
\end{abstract}

\section{Keywords}

Friction, Ice, Roughness, Liquid-Like-Layer, Ice friction model

\section{Introduction}

The study of friction between metals and ice is as struggling as important in a wide range of fields, from ice sports to motorized traffic [1-3]. That being said, the debate behind the origin of the low friction coefficient that characterizes ice surfaces is still open even after decades of experimental and theoretical research on both saline and freshwater ice [4-10].

It is indisputable fact that the friction coefficient of a solid surface sliding on ice is related to the existence of a thin layer of water between the slider and the ice itself. There are three main mechanisms that govern the formation of this layer [10]: surface melting, pressure meltingand frictional melting. The surface melting is a spontaneous generation of a thin layer of melted ice (with thickness in the order of magnitude of few nanometers) without contact with other bodies and without any applied pressure, when the temperature approaches the melting value. The origin of this phenomenon observed in a number of solid surfaces is still 
under debate, although the most prevailing theories indicate the minimization of free surface energy as the main cause [10]. The pressure melting is responsible for lowering the melting temperature of ice by applying a pressure. The frictional melting is generated by the heat dissipated by the friction force; this heat increases the interface temperature, and it is considered as the most relevant mechanism in the formation of water at the interface in sliding systems $[2,11-14]$. The thickness of the water layer defines the lubrication regime of a given sliding system and it is influenced by temperature, normal force and sliding velocity $[10,15,16]$. Consequently, varying the experimental parameters it is possible to explore all lubrication regimes, from boundary to hydrodynamic. According to literature the surface roughness has the same importance since it defines the height of the asperities that interact with each other [10,17-19].

In boundary (or dry) lubrication regime the frictional behavior is governed by the real contact area between the solids, in which adhesion is the main source of friction and heat dissipation [10, 20-22]. In this regime the thickness of the liquid water is indeed very low, in the order of magnitude of few molecular layers [23, 24]. Increasing the sliding velocity, the water layer thickness increases and starts to support the load of the slider; this condition is typical of the mixed lubrication regime. The interfacial conditions of this lubrication regime are not fully clarified yet, and there are different theories about it $[10,25]$. In particular Kietzig et al. [10] assume that mixed lubrication occurs when the temperature at the contact point is greater than the melting temperature of ice, but the thickness of the water layer is still lower than the roughness of the counterpart's surface. In this vision, both solid-solid and lubricated contact coexist at the interface. In contrast, Makkonen et al. [25] assume that at the actual contact point the temperature rises to the melting point, but not over this value. The contact is, therefore, fully lubricated, even with very low thickness of the layer of water, and there is no more solid-solid interaction between the surfaces. All the experimental data reveal a dependence of the Coefficient of Friction $\operatorname{CoF}(\mu)$ on sliding velocity $(v)$ as $\mu \sim 1 / \sqrt{v}$ $[26,27]$.

In the hydrodynamic regime the CoF starts increasing proportionally to $\sqrt{v}$ [28-30]. Kietzig et al. [10] assume that this regime starts when the thickness of the water layer becomes greater than the average roughness of the involved surfaces.

The present work examines the role played by the surface of the slider in terms of roughness and topography, on the friction regimes. Tribological tests of a steel-ice contact are performed, varying temperature and sliding velocity. The dependence of friction on surface morphology is studied by inducing different degrees of roughness on stainless steel surfaces. Finally, an analytical model that directly correlates surface roughness to the friction coefficient is presented and then successfully applied to the experimental results.

\section{Materials and methods}

The ice samples are produced by freezing distilled water in a commercial freezer unit at -8 C. Thin layers of water are frozen on the top of each other in order to minimize cracking and bubbles formation, and to produce a polycrystalline surface mainly exposing basal planes [10]. An optical image of the surface replica is shown in Figure 1. Curved grain boundaries of ice and their peculiar $120^{\circ}$ angles are clearly visible. In figure 1 sublimation pits ("etch pits") are also visible. These spots are created by a higher sublimation speed at location where dislocation slip lines cross the surface $[13,14]$. The ice surface does not go through a long aging process so no frost deposition is visible $[13,14]$. 
The average roughness $\left(R_{a}\right)$ of ice is measured by stylus profilometer on a replica of the surface (prepared using a vinylpolysiloxane-based liquid thermo-polymer) [26], and a value of $100 \pm 10 \mathrm{~nm}$ is observed.

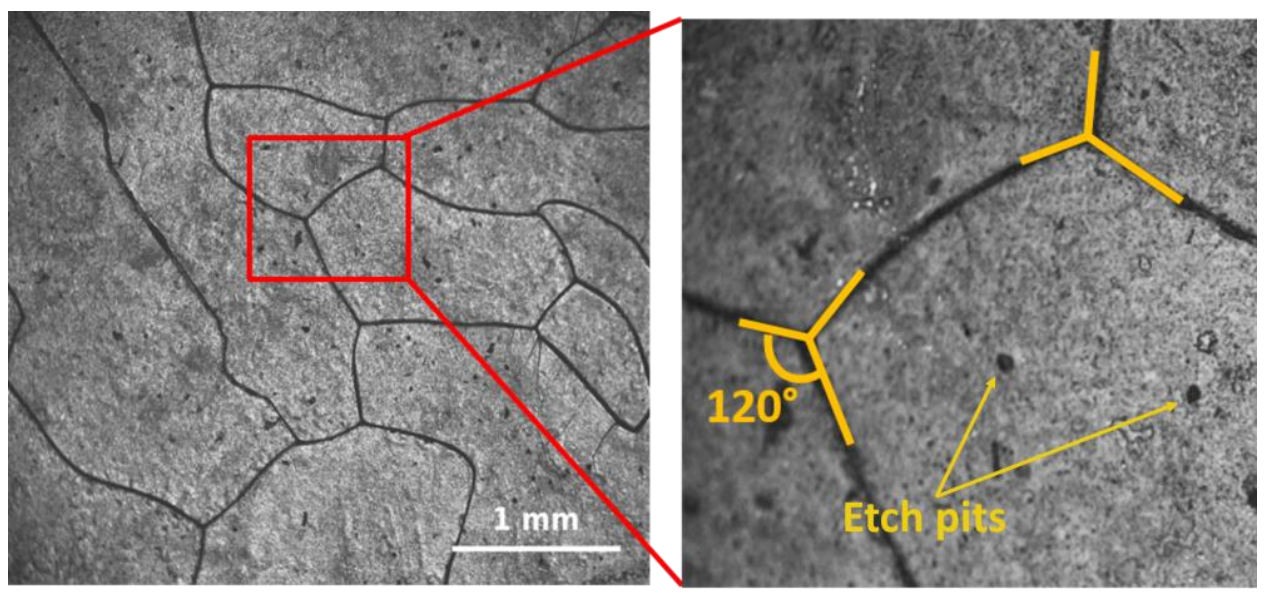

Fig. 1. Optical microscopy image of an ice surface replica. The magnification shows the characteristic $120^{\circ}$ angles formed by the grain boundaries at almost each cross. Etch pits are also visible $[13,14]$

A controlled surface roughness on the stainless-steel pins is induced through mechanical polishing and sand-blasting. Three different pins are produced, labeled \#1, \#2 and \#3. The pin \#1 is polished with alumina slurry (1-3 $\mu$ m diameter), while pins \#2 and \#3 are grinded through sand-blasting (grit 320 and 180 respectively). These techniques ensure a good isotropy of the surface roughness, without introducing preferential directions on the surface. Also in this case, surface morphology is measured through a stylus profilometer (3D profiles are shown in Figure 2). Different parameters are used to characterize the surface roughness (Table 1); all of these parameters show a monotone trend, except for the contact angle which is characterized by the same value (within the experimental error) for each sample. $R_{a}$ and $\mathrm{R}_{\mathrm{dq}}$ of pin \#1 are one order of magnitude lower than those of pin \#2 and \#3. Furthermore the roughness of pin \#1 is almost the same as the one of the ice surface (see below).

\begin{tabular}{ccccccc}
\hline Steel Slider & $\mathrm{R}_{\mathrm{a}}(\mathrm{m})$ & $\mathrm{R}_{\mathrm{dq}}(\mathrm{deg})$. & $\mathrm{S}_{\mathrm{k}}$ & $\mathrm{K}_{\mathrm{u}}$ & $\mathrm{D}$ & Contact angle (deg.) \\
\hline$\# 1$ & $0.11 \pm 0.02$ & $7.3 \pm 1.0$ & $2.35 \pm 0.0$ & $9.3 \pm 0.4$ & $2.763 \pm 0.005$ & $52 \pm 2$ \\
$\# 2$ & $1.4 \pm 0.1$ & $27.2 \pm 1.5$ & $1.2 \pm 0.3$ & $6.4 \pm 1.2$ & $2.359 \pm 0.005$ & $63 \pm 10$ \\
$\# 3$ & $2.6 \pm 0.1$ & $33.6 \pm 1.1$ & $0.9 \pm 0.3$ & $3.4 \pm 0.9$ & $2.276 \pm 0.005$ & $55 \pm 8$ \\
\hline
\end{tabular}

Table 1. Roughness parameters for the stainless-steel pins: average roughness $\left(R_{a}\right)$, RMS slope $\left(R_{d q}\right)$, skewness $\left(\mathrm{S}_{\mathrm{k}}\right)$, kurtosis $\left(\mathrm{K}_{\mathrm{u}}\right)$, fractal dimension (D) and contact angle.

The tribological tests are performed in pin-on-disc configuration on a UMT3-CETR tribometer (http://www.cetr.com/eng/products/umt-3.html) enclosed in a thermally insulated chamber, where the temperature is controlled by a flow of cold dry air. The system is able to reach low temperatures until $-25^{\circ} \mathrm{C}$, with an error of $\pm 1^{\circ} \mathrm{C}$. All the tribological tests are performed with a constant normal load of $15 \mathrm{~N}$, (nominal pressure of $0.085 \mathrm{MPa}$ ). Tests are performed at constant environmental temperature (between $-17^{\circ} \mathrm{C}$ and $-2^{\circ} \mathrm{C}$ ), increasing the sliding velocity in consecutive steps (from $0.025 \mathrm{~m} / \mathrm{s}$ to $1 \mathrm{~m} / \mathrm{s}$ ), each one of 2 minutes of length for a total duration of 16 minutes ( 8 different speeds are tested).

Unfortunately the temperature of the ice cannot be measured. Anyway the ice sample is left sitting in the tribometer chamber for about one hour.. This time should be enough for the ice to reach the same temperature of the surrounding environment. 


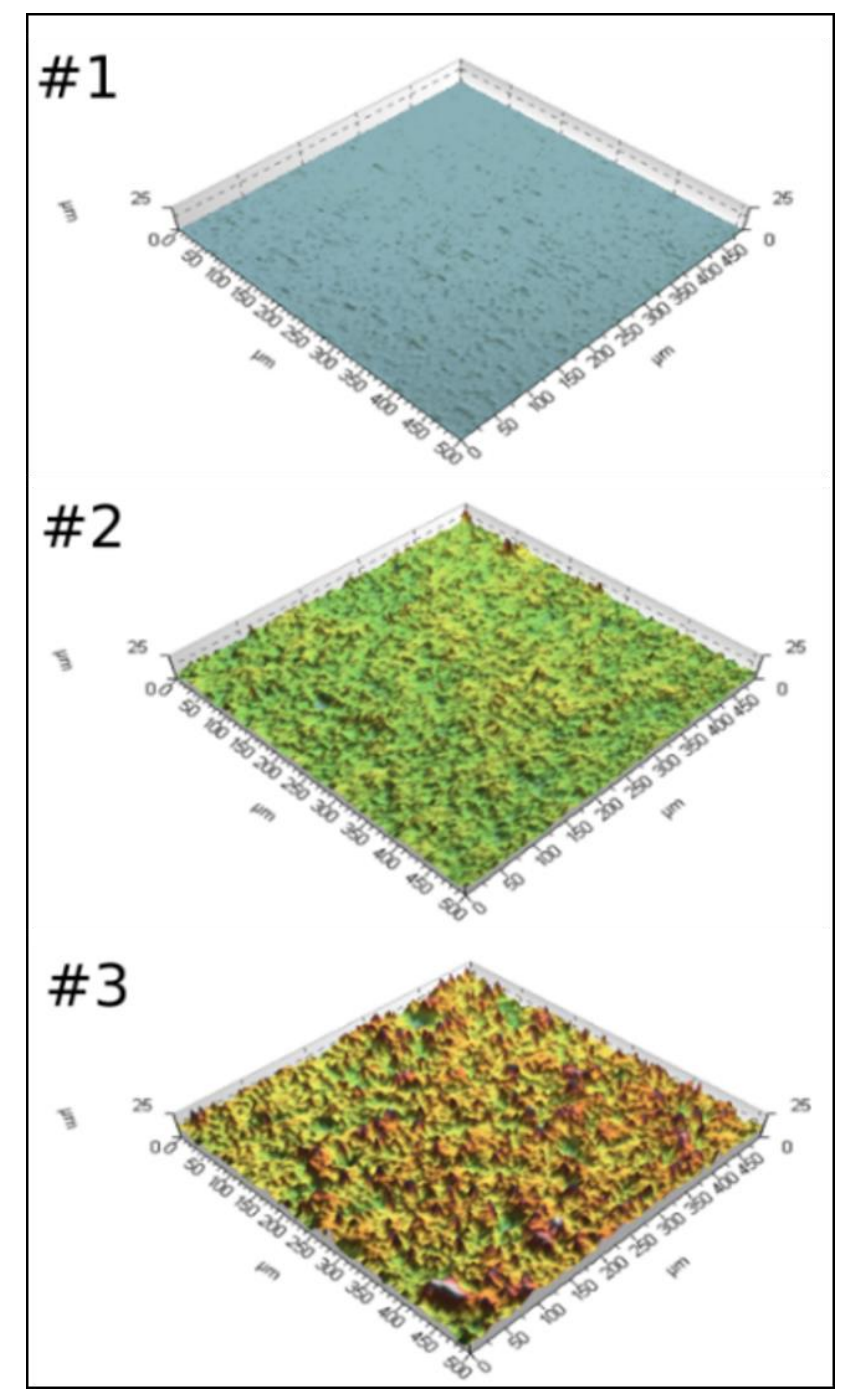

Fig. 2. 3D surface profile of the stainless-steel pins.

To improve the accuracy and reliability of the experimental results and to avoid systematic errors, each test is performed on a freshly prepared ice surface, and four different measures are made for each temperature. The friction values reported here are the average of all of the measures reported in the graphs. At the beginning of each test a short sliding run is made on the ice surface, in order to remove or reduce ice macroscopic asperities that could compromise the stability of the tests. This preliminary run is done taking the pin in contact with the ice disk at a load of $2 \mathrm{~N}$ and rotating the disk 3 times.

\section{Experimental Results}

Values of friction coefficient obtained for steel on ice versus the sliding velocity are shown in Figure 3 for the three pins, at different ambient temperatures between $-2^{\circ}$ and $-17^{\circ} \mathrm{C}$.

The first evidence is that the operating temperature (presumably, the bulk temperature of slider and ice) does not affect significantly the COF value. This is probably due to the experimental setup. Using a pin-on-disc configuration the repeated passages of the pin on the same circular track results in frictional heat dissipation that causes an increase of the temperature at the steel-ice interface. The interface temperature, resulting from the thermal 
equilibrium between the dissipation of frictional heat during the contact and the refreezing between two consecutive passages, saturates at a value larger than the one of the steel, of the ice bulk and of course of the surrounding environment [31].
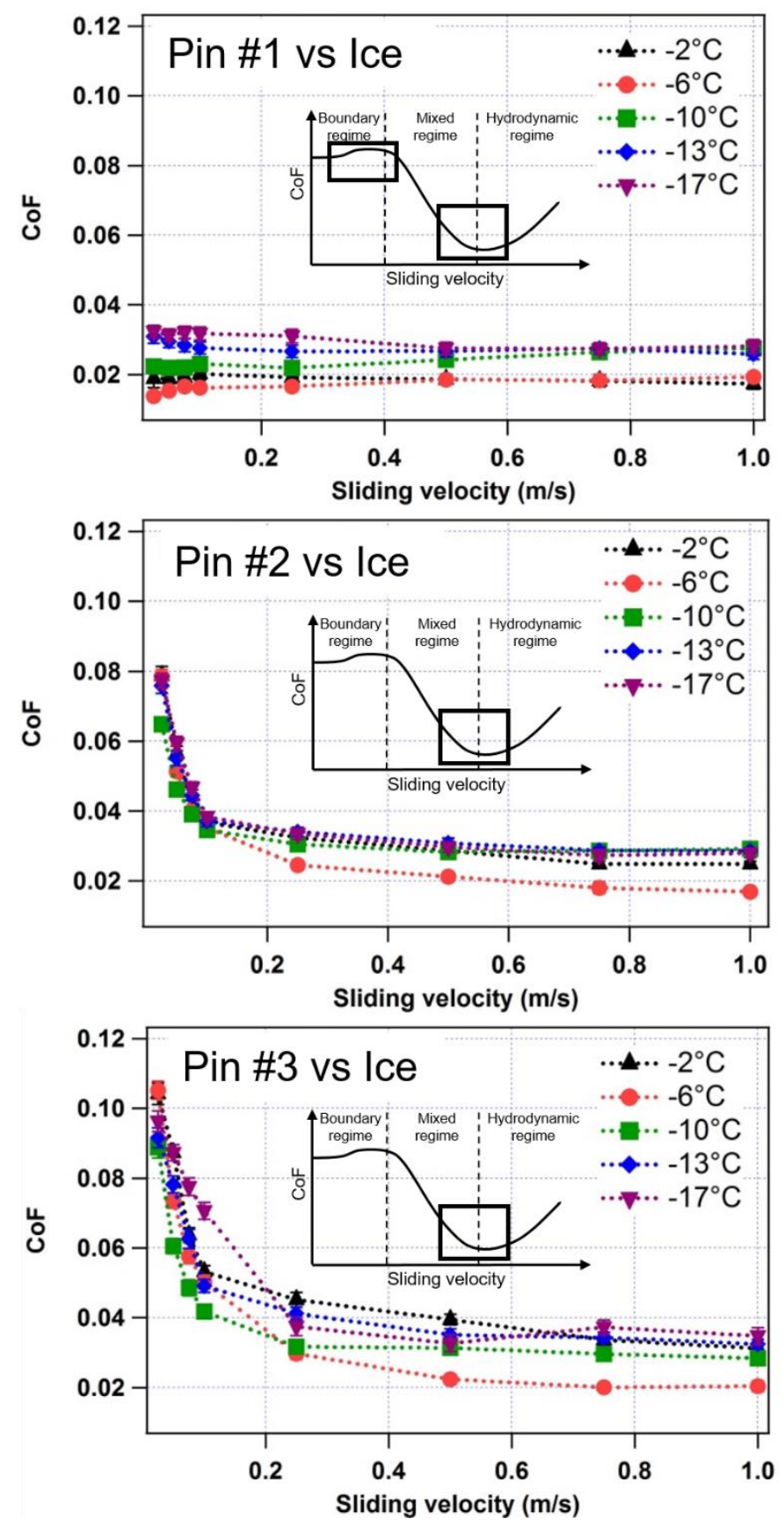

Fig. 3. Friction coefficient results obtained from the pin-on-disc tests. The values are plotted as function of the sliding velocity. The tests are conducted at different temperatures (line colors and dot shapes). The error bars are not clearly visible since their dimension is comparable to the size of the dots.

A quick calculation shows that at a speed of $0.1 \mathrm{~m} / \mathrm{s}(80 \mathrm{rpm})$ the time between two passages over the same spot is less than 1 second. This causes the temperature of the ice surface and of the steel slider surface to increase until balance is reached. So, basically, after a short running-in, the CoF reaches a steady state where both the interface temperature and all the other conditions do not vary. The thickness of the water layer produced by melting in this state is independent from the initial conditions, thanks to the two competitive effects 
contributing to his formation, the frictional melting and the squeeze-out of the water (as stated by [32]).

A second evidence from Fig. 3 is that the frictional behavior is quite different for pin \#1 (the smoothest one) and pin \#2 and \#3, (roughness about 1 order of magnitude larger).

Results from pin \#2 and \#3 show descending COF values as the sliding velocity increases. The descent is initially quite sharp. Between $0.025 \mathrm{~m} / \mathrm{s}$ and $0.3 \mathrm{~m} / \mathrm{s}$ the CoF values move from $0.09 \div 0.11$ (pin \#3) and $0.06 \div 0.08$ (pin \#2) down to $0.03 \div 0.05$. Then, the dependence of $\mathrm{CoF}$ values from the sliding velocity markedly reduces and the friction curve becomes almost flat at a CoF value of $0.02 \div 0.04$. This trend is typical of the transition region between mixed and hydrodynamic friction regimes, as evidenced by the grey squares in the insets in panels 2 and 3 of Fig. 3.

Despite the very similar trends, the accuracy of the measured CoF values enables to be confident on the overall lower values measured with pin \#2 with respect of pin \#3, and to ascribe this difference to the roughness difference (less than a factor 2) between the two steel surfaces.

Results from pin \#1, on the contrary, show a nearly constant CoF (roughly between 0.02 and 0.03 ) along the entire sliding velocity range, indicating that the system is in a different lubrication regime. The assignment to a specific lubrication regime is however quite difficult, because a constant CoF behavior is typical either of the boundary friction regime or of the minimum between the mixed and the hydrodynamic regime (see the grey squares evidenced in the inset of panel 1 of fig. 3). The low values of CoF seem to support the second hypothesis, because low $\mathrm{CoF}$ are typical of the mixed lubrication regime. However, in absence of high wear effects (e.g. ploughing friction or cracking), the low shear stress of ice allows low values of CoF even in the dry lubrication regime. This condition could actually apply in the present case, thanks to the very low roughness of the pin \#1 and to the low applied pressure. The pin has indeed nearly the same roughness of the ice surface, and its low waviness leads to a high real contact area. Furthermore, the roughness profile is quite broaden ( $\mathrm{Rdq}=7.3$, Table 1$)$. These features, coupled with a nominal applied pressure of $0.085 \mathrm{MPa}$ (nearly two order of magnitude below the breaking pressure of ice), suggest that phenomena like ploughing friction and cracking of the ice bulk close to the surface are negligible.

\section{Theoretical Model and Discussion}

\subsection{Ice friction model}

In literature there are several theoretical models about ice tribology [16, 18, 25, 32-34]; each one trying to calculate the CoF of ice, and to cope with the interdependence of the different involved parameters.

One of the most complete models is the one developed by Makkonen [25], where the only source of friction is the shear strength $\tau$ of the water layer, due to its viscosity:

$$
F_{w e t}=\tau A=\frac{\eta v}{h} A
$$

where $A$ is the real contact area, $\eta$ and $h$ are respectively the viscosity and the thickness of the LLL.

Through the calculation of the heat flux generated at the interface by the friction force it is possible to estimate the thickness $h$ of the water layer, produced during the sliding; inserting this expression in Eq. (1) the expression for the coefficient of friction $\mu_{w e t}$ [25] is obtained: 


$$
\mu_{w e t}=\frac{1}{H_{1} \sqrt{a}} \cdot \frac{1}{2 \sqrt{2 v}}\left(\gamma+\sqrt{\gamma+8 \eta L \rho v^{2}}\right)
$$

Where

$$
\gamma=\Delta T_{1} \sqrt{c_{1} \kappa_{1} \rho_{1}}+\Delta T_{2} \sqrt{c_{2} \kappa_{2} \rho_{2}}
$$

and

$$
\Delta T_{i}=T_{i}-T_{m}
$$

\begin{tabular}{|c|c|c|c|c|c|}
\hline \multirow{2}{*}{\multicolumn{2}{|c|}{ List of symbols }} & \multicolumn{4}{|c|}{ Numerical values } \\
\hline & & & Ice & Steel & Water \\
\hline$a$ & Contact length & & & & \\
\hline$T_{i}$ & Temperature $\left({ }^{\circ} \mathrm{C}\right)$ & -17 to -2 & & & \\
\hline$T_{m}$ & Melting temperature of ice $\left({ }^{\circ} \mathrm{C}\right)$ & Ref. [13] & & & \\
\hline V & Sliding velocity $(\mathrm{m} / \mathrm{s})$ & 0.025 to 1 & & & \\
\hline$H_{i}$ & Ice hardness (MPa) & Ref. [13] & & & \\
\hline$i$ & Density $\left(\mathrm{Kg} \mathrm{m}^{-3}\right)$ & & 916 & 7750 & \\
\hline & Water density at $0^{\circ} \mathrm{C}\left(\mathrm{Kg} \mathrm{m}^{-3}\right)$ & & & & 1000 \\
\hline & Water viscosity at $0^{\circ} \mathrm{C}\left(\mathrm{Kg} \mathrm{m}^{-1} \mathrm{~s}^{-1}\right)$ & & & & $1.76 \cdot 10^{-3}$ \\
\hline$i$ & Thermal conductivity $\left(\mathrm{W} \mathrm{m}^{-1} \mathrm{~K}^{-1}\right)$ & & 2.2 & 20 & \\
\hline$c_{i}$ & Specific heat $\left(\mathrm{J} \mathrm{Kg}^{-1} \mathrm{~K}^{-1}\right)$ & & 2090 & 460 & \\
\hline$L$ & Water latent heat $\left(\mathrm{J} \mathrm{Kg}^{-1}\right)$ & & & & $330 \cdot 10^{3}$ \\
\hline
\end{tabular}

A list of the symbols used in the previous part is reported in Table 2.

Table 2. List of used symbols and their numerical values; $\mathrm{i}=1$ for ice, $\mathrm{i}=2$ for slider. The contact length $a$ is calculated from data analysis in the next section.

In this model the role of the surface morphology is poorly included, as it enters only in the definition of $a$, which is defined as the characteristic length of the real area of contact between the ice and the slider, and it is fixed to $a=1 \mathrm{~mm}$. With such a great contact length the dependence from the roughness of the slider is not included in the model, and only the lowfrequency waviness of the surface affects the tribological behavior of the system.

In order to include the contribution of the surface roughness to the tribological behavior of the system a suitable analytical model is developed and successfully applied to the present case.

\subsection{Surface roughness}

The theoretical model for anisotropic friction developed here considers the sliding motion between rough surfaces and directly correlates the effects of different roughnesses to the friction coefficient. This model is based on the one introduced by Mroz and Stupkiewicz [35], in which the contact between two surfaces is modelled by a set of springs with only longitudinal compliance. In the present work two orthotropic surfaces with general asperities are considered (Figure 4a).

During the sliding of one surface on the other, the vertical springs have to accommodate the movement modifying their length, being compressed or elongated. This length variation is 
reflected on the elastic forces that the two surfaces shall exchange. Furthermore the moving surface can change the direction of sliding ( $v_{0}$ is the sliding velocity), thus it could be or not be perpendicular to the asperities of the fixed surface (Figure $4 \mathrm{~b}$ ).

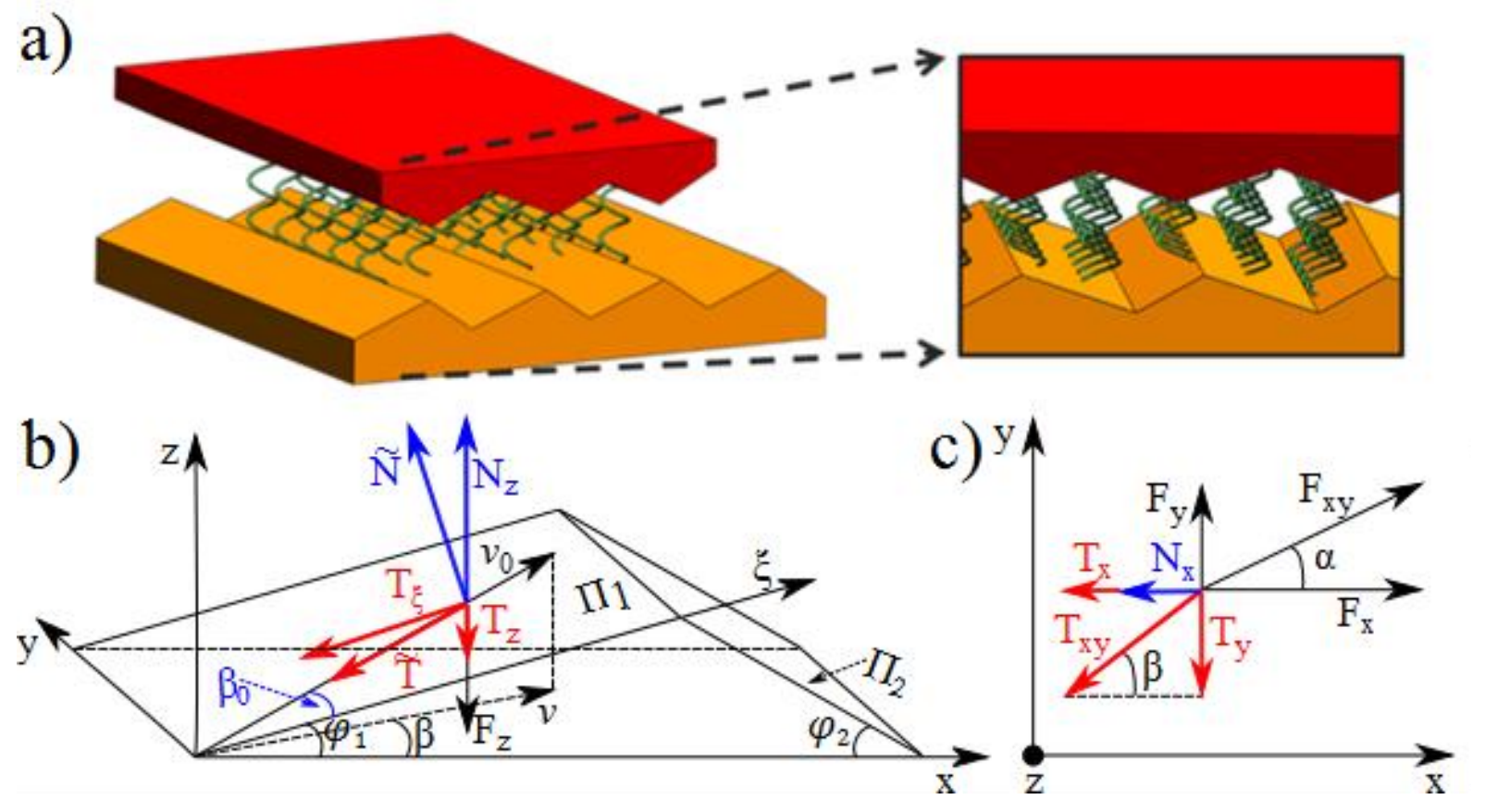

Fig. 4. a) A sketch of two orthotropic surfaces with general asperities as considered in the theoretical model; the interaction between them is modeled by a set of springs with only longitudinal compliance; b) single wedge asperity in which the trajectory of the sliding velocity is shown: on the $\Pi 1$ plane (v0) and in the xyplane $(\mathrm{v})$; $\mathrm{c}$ ) the decomposition along the $\mathrm{z}$-axis of the acting forces due to a single spring and the reacting vertical for $\mathrm{F}$.

If $R_{z}$ is the global reaction force along the $z$-axis and $R_{x, y}$ is the global reaction force acting on the $x y$-plane (both due to all of the springs and averaged on every single wedge asperity), we can express the total coefficient of friction as follows:

$$
f=\frac{R_{x, y}}{R_{z}}=\frac{R_{x}}{R_{z}} \frac{1}{\cos \alpha}=\frac{R_{y}}{R_{z}} \frac{1}{\sin \alpha}
$$

Where

$$
\tan \alpha=\frac{R_{y}}{R_{x}}=(\cos \varphi)^{2} \tan \beta
$$

Considering only the effect of a single spring, $\alpha$ is the angle reported in Figure 4c.

To evaluate this friction coefficient (Eq. 5), it is necessary to determine the reaction forces in the global reference system $x y z$, starting at first by analyzing the contribution of a single spring. All the details of the calculation are reported in Appendix.

This model considers the local interaction between the two surfaces through the introduction of a generic local Coefficient of Friction (named $\mu$ ), which does not consider any morphological effect and does not specify any physical mechanism at its base. In order to contextualize the model to the present specific case, the ice friction model [25] previously described is chosen, and therefore the generic local $\operatorname{CoF} \mu$ is substituted with $\mu_{w e t}$, which is the coefficient we obtained from Equation (2). 


\subsection{Application of the model to the experimental data}

To be able to apply this general model to the tribological system reported in this work, two particular cases are selected; in both cases the asperity is considered symmetrical, by taking $\varphi_{1}=\varphi_{2}=\varphi$. The asperity slope is referring to the pins roughness, thus it is assumed equal to Rqd, one of the parameters which we obtained from a stylus profilometer. This choice matches the roughness data obtained from the profilometer.

The first case considers the sliding motion to occur only along the $x$ direction, by taking $\alpha=$ 0 . In this situation the sliding appears to be against the wedge asperities, and $\mathrm{CoF}$ is maximum. On the contrary, the second case assumes the motion along $y$ direction, with $\alpha=$ $\pi / 2$, leading to the minimum CoF.

The expressions for these two cases (full calculations are reported in Appendix) are:

$$
\begin{gathered}
f^{u}=f_{x}=\frac{\mu_{w e t}}{1-(\sin \varphi)^{2}\left(1+\mu_{w e t}{ }^{2}\right)} \\
f^{l}=f_{y}=\frac{\mu_{w e t}}{\cos \varphi}
\end{gathered}
$$

where $f^{u}$ and $f^{l}$ are respectively the upper and the lower limit of the CoF. All the possible orientations of the sliding motion are thus automatically considered, and the real value of the $\mathrm{CoF}$ is included between these upper and lower limits.

The slope of the asperity reported in the model can be approximated with the $R_{\mathrm{dq}}$ of the profile, reported in Table 1, while the local CoF in Eq. (7) is substituted by expression (2). Since the shear strength of a liquid layer is the only source of friction in this picture, a further hypothesis must be introduced: the whole real contact area has to be covered by a water layer, and the contact during the sliding motion must be fully lubricated. This hypothesis does not exclude the presence of ploughing by the slider asperities into the ice, but this ploughing is expected to be mediated by a thin layer of water.

Furthermore, the theoretical model describes the motion as linear while friction experiments are performed in pin-on-disc configuration. In fact one of the hypothesis of the model is that the slider always runs on a new ice surface, whose surface temperature is the same of the bulk. In the rotational real case, instead, the slider performs repeated passages over every point of the ice track periodically, with a short refreezing time due to the short radius of the track and to the sliding speed. With these assumptions the interface temperature of the ice rises, since every passage of the slider dissipates frictional heat. After some passages the temperature at the interface can be considered to be homogenous and close to the melting point of ice. For these reasons the terms $T_{1}$ and $T_{2}$ from Eq. (3) can be neglected. This assumption is justified also by the very weak temperature dependence of the tribological tests shown in Figure 3.

Although the interface temperature is taken as homogenous at melting point of ice the bulk temperature is absolutely not and neither is the hardness of the ice. This parameter is still calculated with the bulk temperature since the depth of the stress due to the indentation of micrometric asperities is expected to be in the order of few microns, while the melting process affects a much lower thickness very close to the interface. The values of ice hardness used in this paper are based on the work of Makkonen et al. [25], following the relation

$$
H_{i}=C_{1} T+C_{2}
$$

with $C_{1}=-5.08 \mathrm{MPa} / \mathrm{K}$ and $C_{2}=15.19 \mathrm{MPa}$. 
It is now possible to fit the experimental data with both expressions (7) and (8), using the contact length $a$ as the only fitting parameter. The results of this procedure are shown in Figure 5, where the confidence parameters $r$ of the "blue" fit are also reported, together with the values of the fitting parameter $a$ for pin \#2 and \#3.
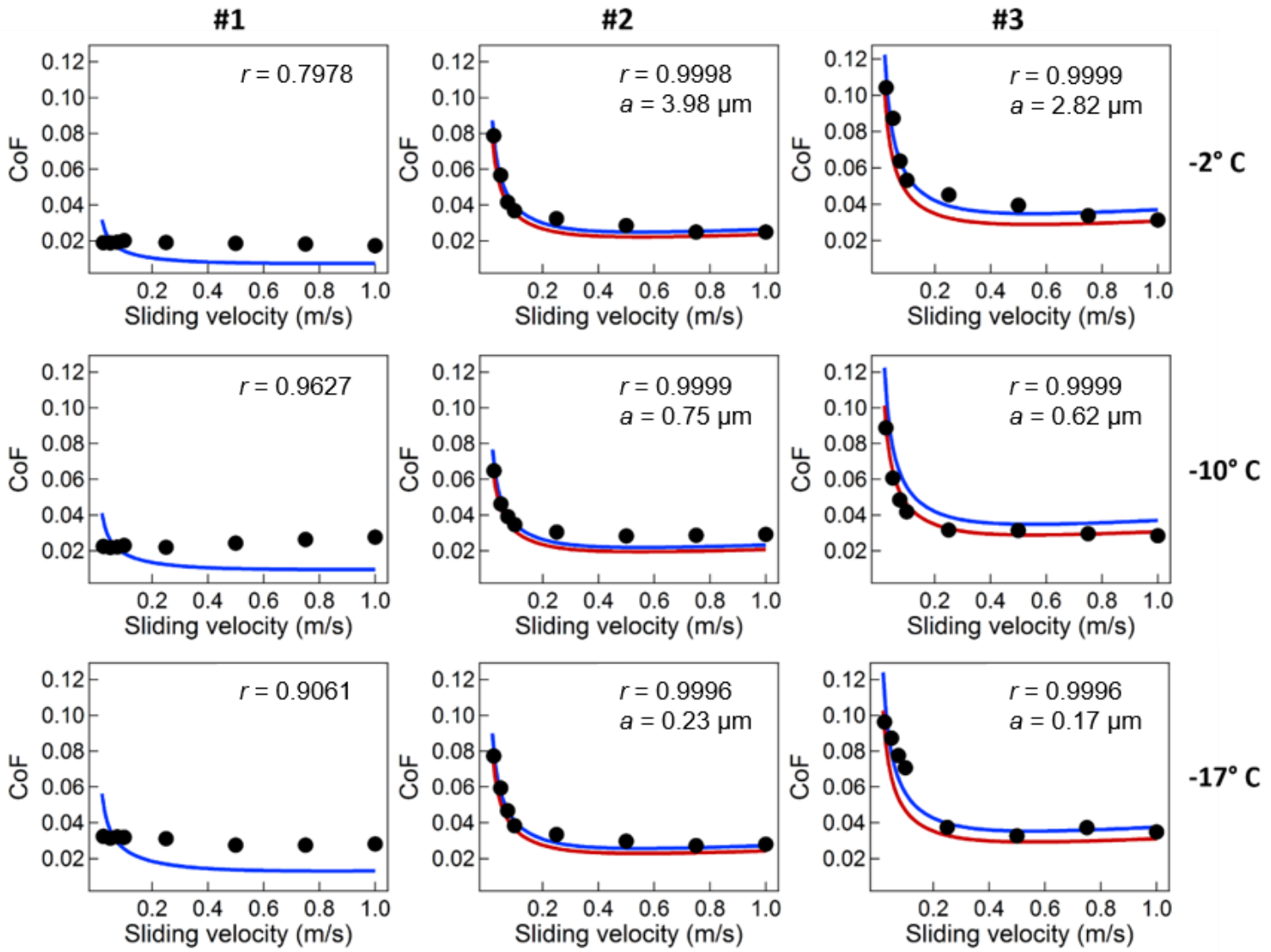

Fig. 5. Best fits of the experimental data obtained with fitting functions $f^{u}$ (blue line) and $f$ (red line) for three selected temperature $\left(-2^{\circ},-10^{\circ}\right.$ and $\left.-17^{\circ} \mathrm{C}\right)$. The values of the confidence parameter $r$ of the "blue" fit are also reported, together with the values of the fitting parameter $a$ for pin \#2 and \#3.

The first evidence is that the model fits quite well the experimental results obtained with pins \#2 and \#3. In the explored range of sliding velocities, both the trends and the absolute values of $\mathrm{CoF}$ are satisfactorily described by the model, with a confidence parameter $r$ ranging between 0.9996 and 0.9999 . On the contrary, the model does not fit satisfactorily the experimental results obtained with pin \#1, in particular does not account for the almost constant CoF value measured for all the sliding velocities explored.

It must be outlined that the model introduces the shear stress of the liquid like layer as the only source of friction, therefore its ability to fit results from pins to \#2 and \# 3 confirms that these sliders operate in a lubricated regime.

On the other hand, the model failure in describing the experimental results from pin \#1 suggests that in this case the system experiences a different lubrication regime. The low roughness of pin \#1 compared to pins \#2 and \#3 is expected to induce a lower frictional interface heat and consequently a reduced frictional melting. Being the frictional melting considered as the most relevant mechanism in the formation of the water layer in sliding systems [11], pin \#1 is expected to work in an almost dry lubrication regime.

This description, that assigns a key role to the interface heating during the sliding and outline the contribution of the interface roughness in determining the interface temperature, can be 
confirmed by exploring another relevant parameter that affect the interface temperature, namely the thermal conductivity of the slider.

Preliminary tests were performed with a pin made of hard phenolic resin. From the mechanical point of view the resin is softer than steel (by a factor 10), but it is still much harder than ice (by a factor close to 15). All the hypothesis on the contact mechanic of the system are still valid, in particular the contact area only depends on the softer material at the interface (the ice). From the thermal point of view, however, a conductor (steel has a thermal conductivity of about $20 \mathrm{~W} / \mathrm{mK}$ ) is replaced by an insulator (resin has a thermal conductivity of about $0.2 \mathrm{~W} / \mathrm{mK}$ ).

The resin pin is prepared with a roughness $R_{a}$ of $0.13 \mu \mathrm{m}$, very close to the roughness of steel pin \#1, and the CoF measurements are performed in the same experimental conditions used for the experiments with steel sliders.

The CoF values obtained at $\mathrm{T}=-2^{\circ} \mathrm{C}$ are reported in Fig. 6, where the corresponding data from steel pin \#1 are also shown for comparison. Results from resin pin show descending CoF values as the sliding velocity increases, indicating a clear transition to a mixed lubrication regime thank to the formation of a water layer associated to the higher interface temperature.

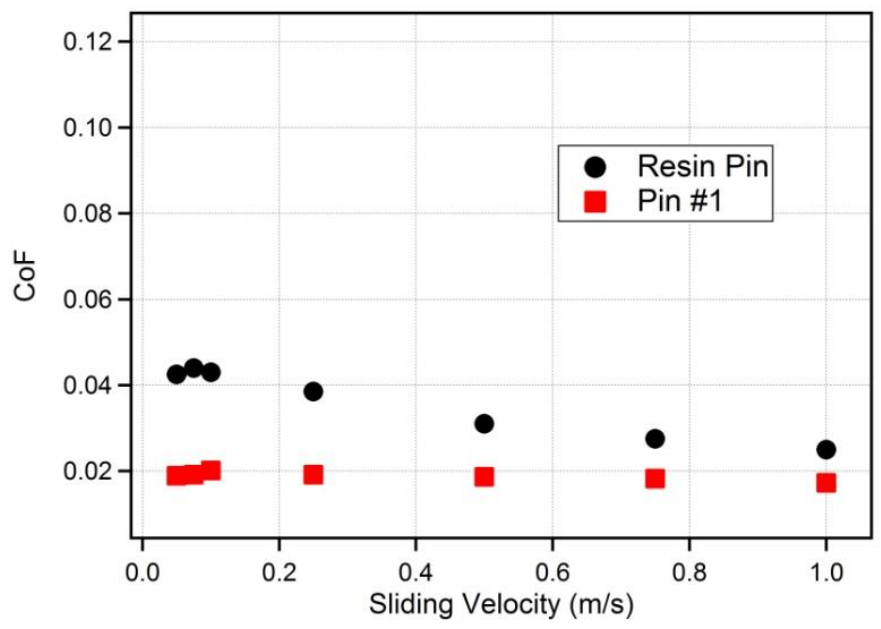

Fig. 6. Comparison between the friction coefficient results obtained from the resin pin tests performed at $-2^{\circ} \mathrm{C}$ (black dots) and the results from the steel pin \#1 tests (red dots) performed at the same temperature. The values are plotted as function of the sliding velocity.

\subsection{Actual and nominal contact area}

In order to compare the results of a obtained with the fitting procedure, a realistic value of the contact length has to be evaluated. For this purpose the topography of the surface has been approximated with a regular pattern of triangular asperities (like the one sketched in Figure 7 on the right). Such sort of profile shows the same $R_{d q}$ of the real random surface used in the experimental tests. When that surface is pressed with a load $F_{z}$ on a flat ice surface, the tips of the asperities penetrate into the ice, both through melting and elastoplastic deformations. The real contact area $\left(A_{\text {real }}\right)$ is inversely proportional to the hardness of the ice $H_{1}$ [25], while the nominal contact area $\left(A_{\text {nom }}\right)$ is inversely proportional to the applied pressure $\sigma$ :

$$
A_{\text {real }}=\frac{F_{z}}{H_{1}} \quad A_{\text {nom }}=\frac{F_{z}}{\sigma}
$$

It is therefore possible to write the ratio $\Delta A$ which represents the ratio between the nominal and real contact area: 


$$
\Delta A=\frac{A_{\text {nom }}}{A_{\text {real }}}=\frac{H_{1}}{\sigma}
$$

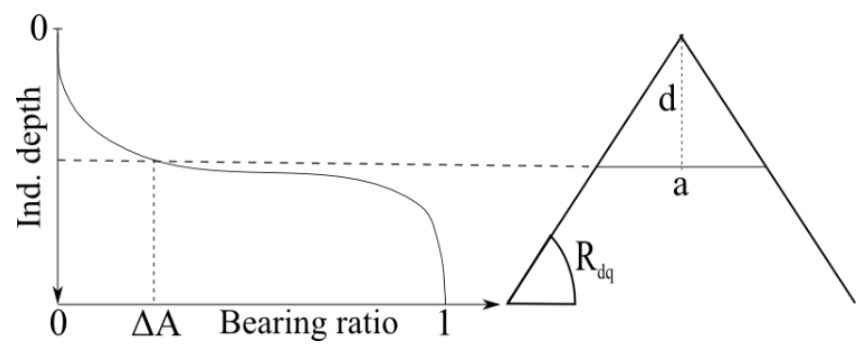

Fig. 7. Correlation between the model surface and the bearing ratio curve.

By taking the bearing-ratio curve of the real surface it is possible to correlate $\Delta A$ with the average indentation depth of the triangular asperities (see Figure 6). With simple geometrical calculations an average value of the contact area length at the interface is found:

$$
a=2 \frac{d}{\tan R_{d q}}
$$

As shown in Figure 8 there is good agreement between the values obtained from the fits (section 4.2) and from the bearing ratio curve, especially at the lower temperatures. Since we have considered the ice as a flat surface, and the pin material is not soft, the value of a was expected to be of the same order of magnitude as Ra, due to low normal load and thus small plastic deformations.

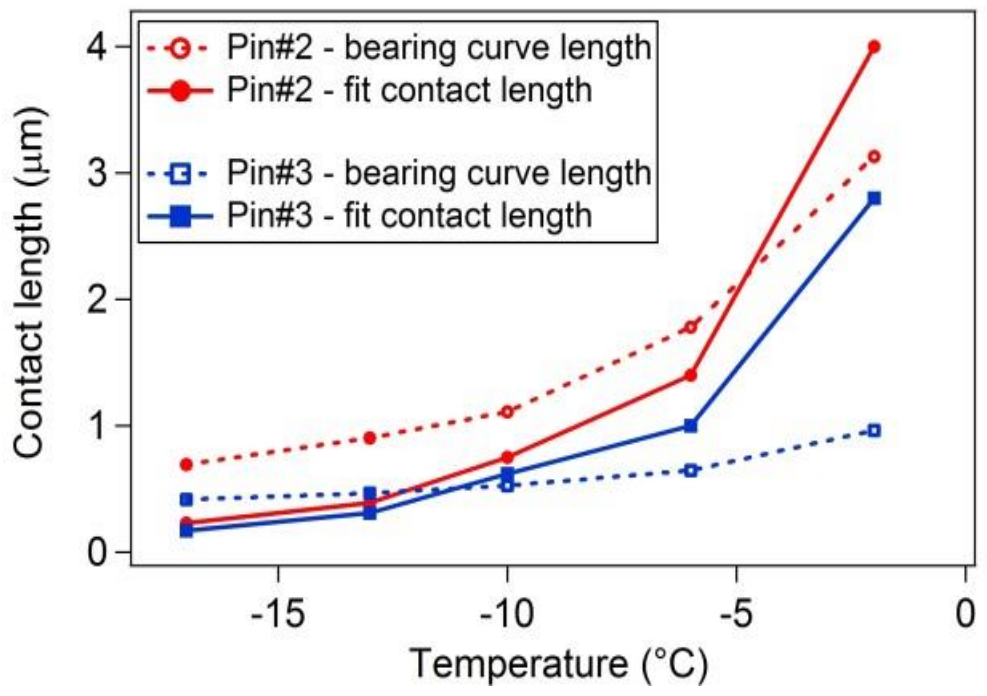

Fig. 8. Fits agreement.

\section{Conclusions}

The influence of surface morphology on the CoF of a steel-ice interface is studied both experimentally and theoretically with the purpose to clarify the tribological behavior of ice. Three different degrees of random roughness are induced on stainless-steel surfaces sliding on ice in a pin-on-disc configuration, one being comparable to the roughness of the ice and the other two one order of magnitude higher. Both the temperature of the system and the sliding velocity are varied in a wide range. 
It is shown that surface morphology influences the tribological regime of the system. In the boundary regime the higher the roughness the higher is the CoF. Increasing the sliding velocity (and thus the thickness of the water layer) the role of the interlocking asperity contacts become less relevant, and the roughness has a lower influence on the CoF.

The experimental results are explained by a theoretical model that takes into account the solid contact between two sliding asperities and describes the local CoF between them in terms of shear stress of the water originated from the melting of ice at the interface. The only unknown parameter, the real contact area, is estimated through the bearing-ratio curve and the roughness parameters of the steel surfaces. The good fit between the model and the experimental data obtained with the two rougher sliders confirms the validity of the model and of the physical hypothesis about the mechanism of sliding friction on ice.

The two roughest pins clearly work in a mixed lubrication regime (in the range of tested velocities) while the smoothest pin has a different behavior that cannot be fitted with the model in its current status of development. The water layer behavior and the asperity interaction must be furtherly investigated at this specific value of roughness in order to improve the theoretical model. The trend of the CoF of the smoothest pin is clarified performing the same tests with a pin made of resin with the same surface roughness. This resin pin shows a mixed lubrication behavior. Since the thermal conductivity of the resin is much lower than the one of steel we can conclude that the steel pin with the smoothest roughness work in boundary lubrication regime.

Therefore the interplay between the surface roughness and the thermal conductivity of the counterpart of the ice surface determines the range of applicability of the proposed model.

\section{Acknowledgements:}

N.M. Pugno is supported by the European Research Council [ERC StG Ideas 2011 BIHSNAM n. 279985, ERC PoC 2013 KNOTOUGH n. 632277, ERC PoC 2015 SILKENE nr. 693670], by the European Commission under the Graphene Flagship [WP14 "Nanocomposites", n. 604391].

D. Marchetto and S. Valeri gratefully acknowledge the support by Cost Action MP1303 "Understanding and controlling nano and mesoscale friction". 


\section{References}

[1] A. Roberts, J. Richardson, Interface study of rubber-ice friction, Wear, 67 (1981) 55-69.

[2] D. D. Higgins, B. A. Marmo, C. E. Jeffree, V. Koutsos, J. R. Blackford, Morphology of ice wear from rubber-ice friction tests and its dependence on temperature and sliding velocity, Wear, 265 (2008) 634-644.

[3] S. Colbeck, A. Thorndike, I. Whillans, S. Hodge, S. F. Ackley und G. D. Ashton, Snow and ice, Reviews of Geophysics, 13 (1975) 435-441.

[4] E. M. Schulson, and A. L. Fortt, Friction of ice on ice, Journal of Geophysical Research: Solid Earth 117, no. B12 (2012).

[5] B. Lishman, P. Sammonds and D. Feltham, A rate and state friction law for saline ice, Journal of Geophysical Research: Oceans 116, no. C5 (2011).

[6] S. Jacobsen, G. W. Scherer, and E. M. Schulson, Concrete-ice abrasion mechanics, Cement and Concrete Research 73 (2015) 79-95.

[7] D. C. Hatton, P. R. Sammonds and D. L. Feltham, Ice internal friction: Standard theoretical perspectives on friction codified, adapted for the unusual rheology of ice, and unified, Philosophical Magazine 89, no. 31 (2009) 2771-2799.

[8] C. Wallen-Russell and B. Lishman, The friction of saline ice on aluminium, Advances in Tribology 2016 (2016) ID 1483951.

[9] E. M. Schulson, Low-speed friction and brittle compressive failure of ice: fundamental processes in ice mechanics, International Materials Reviews 60, no. 8 (2015) 451-478.

[10] A. Kietzig, S. Hatzikiriakos, P. Englezos, Physics of ice friction, Journal of Applied Physics, 107 (2010) 081101.

[11] F. P. Bowden, T.P. Hughes, The mechanism of sliding on ice and snow, Proc. R. Soc. London, A, 172 (1939) 280-298.

[12] H. Nyberg, S. Alfredson, S. Hogmark, S. Jacobson, The asymmetrical friction mechanism that puts the curl in the curling stone, Wear 301 (2013) 583-589,

[13] A. Kristona, N. A. Isitmana, T. Fülöpa, A. J. Tuononen, Structural evolution and wear of ice surface during rubber-ice contact, Tribology International 93 (2016) 257-268,

[14] T. Fülöp, A. J. Tuononen, Evolution of ice surface under a sliding rubber block, Wear 307 (2013) 52-59.

[15] C. Stamboulides, P. Englezos und S. G. Hatziriakos, The ice friction of polymeric substrates, Tribology International, 55 (2012) 59-67.

[16] C. Klapproth, T. M. Kessel, K. Wiese, B. Wies, An advanced viscous model for rubberice-friction. Tribology International 99, (2016) 169-181.

[17] S. Sukhorukov and A. Marchenko, Geometrical stick-slip between ice and steel. Cold Reg. Sci. Technol. 100 (2014) 8-19.

[18] A. Kietzig, S. Hatzikiriakos, P. Englezos, Ice friction: The effects of surface roughness, structure, and hydrophobicity, J Appl Phys 106 (2009) 024303.

[19] S. Ducreta, H. Zahouani, A. Midol, P. Lanteri, T.G. Mathia, Friction and abrasive wear of UHWMPE sliding on ice, Wear 258 (2005) 26.

[20] B. Persson, Sliding Friction, Physical Principles and Applications, NanoScience and Technology, Springer, 2000. 
[21] F. Bowden, D. Tabor, The friction and lubrication of solids, 3rd Hrsg., New York: Oxford University Press, 2001.

[22] L. Makkonen, A thermodynamic model of sliding friction, AIP Advances, 2 (2012) 012179.

[23] F. Petrenko, R. Whitworth, Physics of Ice, New York: Oxford University, 1999.

[24] L. Makkonen, Surface melting of Ice, J. Phys. Chem. B., 101 (1997) 6196-6200.

[25] L. Makkonen, M. Tikanmäki, Modeling the friction of ice, Cold Regions Science and Technology, 102 (2014) 84-93.

[26] L. Baurle, U. Kaempfer, D. Szabo und N. Spencer, Sliding friction of polyethylene on snow and ice: Contact area and modeling, Cold Regions Science and Technology, 47 (2007) 276-289.

[27] B. Marmo, J. Blackford, C. E. Jeffree, Ice friction, wear features and their dependence on sliding velocity and temperature, Journal of Glaciology, 51 (2005) 391-398.

[28] F. Albracht, On the influences of friction on ice, Materialwiss. Werkstofftec, 35 (2004) 620.

[29] S. Jones, Friction of melting ice, Ann. Glaciol., 19 (1993) 7-12.

[30] J. de Koning, Ice friction during speed skating, J. Biomech., 25 (1992) 565-571.

[31] J. Dash, Theory of a tribometer experiment on ice friction, Scripta Materialia, 49 (2003) 1003-1006.

[32] S. Colbeck, The kinetic friction of snow, Journal of Glaciology, 34 (1988) 78-86.

[33] P. Oksanen, J. Keinone, The mechanism of friction of ice, Wear, 78 (1982) 315-324.

[34] D. Evans, J. Nye, K. Cheeseman, The Kinetic Friction of Ice, Proc. R. Soc. London A, 347 (1976) 793.

[35] Z. Mroz, S. Stupkiewicz, An anisotropic friction and wear model, Int. J. Solid Structures, 31 (1994) 1113-1131. 


\section{Appendix}

The full calculations of the theoretical model previously exposed are reported in this section.

Referring to Figure $6 \mathrm{~b}$, the normal force $\widetilde{N}$, that is the force perpendicular to the $\Pi_{1}$ plane (tilted by an angle $\varphi_{1}$ with respect to $x y$-plane), and the tangential force $\tilde{T}$ (where $\mu$ is the local coefficient of friction) acting on $\Pi_{1}$ are equilibrated by the reaction forces $F_{x}$ and $F_{y}$ on the $x y$-plane and (Figure 6a) by $F_{z}$ in the vertical direction. From the equilibrium the following equations are obtained:

$$
\begin{gathered}
F_{z}=\widetilde{N} \cos \varphi_{1}-T_{\xi} \sin \varphi_{1} \\
F_{x}=\widetilde{N} \sin \varphi_{1}-T_{\xi} \cos \varphi_{1} \\
F_{y}=\tilde{T} \sin \beta_{0}
\end{gathered}
$$

Where $T_{\xi}=\tilde{T} \cos \beta_{0}$ and from the geometry sketched in Figure 6a:

$$
\tan \beta_{0}=\tan \beta \cos \varphi_{1}
$$

Following the friction law by Coulomb, the tangential force is expressed by:

$$
\tilde{T}=\mu \widetilde{N}
$$

where $\mu$ is the local coefficient of friction. The elastic force due by a single spring is:

$$
F_{z}=K u
$$

where $K$ is the spring stiffness and $u$ the spring displacement; $u$ can be generated by a compressive force (spring compression) or by a tensile force (spring elongation); a spring elongation, means that one surface is separating from the other, so it is assumed that the interaction force is zero.

Referring to a generic plane $\Pi$, tilted by any angle $\varphi$, from Eq. (13), (17) and (18) the expression for $\widetilde{N}$ is obtained:

$$
\widetilde{N}=\frac{K u}{\cos \varphi-\frac{\mu \tan \varphi \cos \beta}{\sqrt{1+\tan ^{2} \varphi \cos ^{2} \beta}}}
$$

The denominator should be different from zero, leading to have $\varphi \neq \frac{\pi}{2}$ and $\mu \neq \cos \varphi$. In particular, in order to have a positive coefficient of friction, it must be $\mu<\cot \varphi$.

Then putting Eq. (16), (17), (18) and (19) in (14) and (15), the reaction forces acting in $x$ and $y$ directions are: 


$$
\begin{gathered}
F_{x}=F_{z} \frac{\sin \varphi \sqrt{1+\tan ^{2} \varphi \cos ^{2} \beta}+\mu \cos \beta}{\cos \varphi \sqrt{1+\tan ^{2} \varphi \cos ^{2} \beta}-\mu \tan \varphi \cos \beta}=F_{z} H_{x}(\mu, \varphi, \beta) \\
F_{y}=F_{z} \frac{\mu \sin \beta}{\cos \varphi \sqrt{1+\tan ^{2} \varphi \cos ^{2} \beta}-\mu \tan \varphi \cos \beta}=F_{z} H_{y}(\mu, \varphi, \beta)
\end{gathered}
$$

Since a certain number $n$ of springs is acting on one wedge asperity, formed by $\Pi_{1}$ and $\Pi_{2}$ plane (respectively tilted by $\varphi_{1}$ and $\varphi_{2}$ with respect to $x y$-plane), the normal elastic force $R_{z}$ ( $z$-direction) is the sum of the resulting vertical forces acting on every plane, thus it is:

$$
R_{z}=\frac{\lambda}{1+\lambda} F_{z}^{(1)}+\frac{1}{1+\lambda} F_{z}^{(2)}
$$

Where $F_{z}^{(1)}$ is the mean normal force acting on a plane tilted by $\varphi_{1}$ with respect to $x y, F_{z}^{(2)}$ is the mean normal force acting on a plane tilted by $\varphi_{2}$ with respect to $x y$ and $\lambda=\frac{\tan \varphi_{2}}{\tan \varphi_{1}}$.

$R_{x}$ and $R_{y}$ are obtained starting from Eq. (22) and putting similarly Eq. (20) or (21):

$$
\begin{array}{ll}
R_{x}^{(1)}=R_{z} H_{x}\left(\mu, \varphi_{1}, \beta\right) & R_{x}^{(2)}=R_{z} H_{x}\left(\mu,-\varphi_{2}, \beta\right) \\
R_{x}^{(1)}=R_{z} H_{x}\left(\mu, \varphi_{1}, \beta\right) & R_{x}^{(2)}=R_{z} H_{x}\left(\mu,-\varphi_{2}, \beta\right)
\end{array}
$$

Thus the expressions for $R_{x}$ and $R_{y}$ become:

$$
\begin{aligned}
R_{x} & =R_{z}\left[\frac{\lambda}{1+\lambda}\left(\frac{\sin \varphi_{1} \sqrt{1+\tan ^{2} \varphi_{1} \cos ^{2} \beta}+\mu \cos \beta}{\cos \varphi_{1} \sqrt{1+\tan ^{2} \varphi_{1} \cos ^{2} \beta}-\mu \tan \varphi_{1} \cos \beta}\right)\right. \\
& \left.+\frac{1}{1+\lambda}\left(\frac{-\sin \varphi_{2} \sqrt{1+\tan ^{2} \varphi_{2} \cos ^{2} \beta}+\mu \cos \beta}{\cos \varphi_{2} \sqrt{1+\tan ^{2} \varphi_{2} \cos ^{2} \beta}+\mu \tan \varphi_{2} \cos \beta}\right)\right] \\
R_{y}= & R_{z}\left[\frac{\lambda}{1+\lambda}\left(\frac{\mu \sin \beta}{\cos \varphi_{1} \sqrt{1+\tan ^{2} \varphi_{1} \cos ^{2} \beta}-\mu \tan \varphi_{1} \cos \beta}\right)\right. \\
& \left.+\frac{1}{1+\lambda}\left(\frac{\mu \sin \beta}{\cos \varphi_{2} \sqrt{1+\tan ^{2} \varphi_{2} \cos ^{2} \beta}+\mu \tan \varphi_{2} \cos \beta}\right)\right]
\end{aligned}
$$

Following the Coulomb's law [20], the tangential force which opposes the sliding motion is proportional to the normal force applied to the surface. This proportion is expressed by the coefficient of friction $f$.

$$
f=\frac{R_{x, y}}{R_{z}}
$$

As previous reported, combining Eq. (21) and (22) with Eq. (25) or (26), expression (5) is found.

The relative motion of one surface with respect to the other can have a generic orientation, so it could happen that the angle $\beta$ (and consequently $\alpha$ ) is 0 or $\frac{\pi}{2}$. 
If $\alpha=0$ and $\beta=0$ the motion is along the $x$-axis and the resultants of the applied forces are only in $\mathrm{x}$ and $\mathrm{z}$ directions $\left(R_{y}=0\right)$. This means that the coefficient of friction assumes a simplified expression, equal to:

$$
\begin{gathered}
f=f_{x}=\left[\frac{\lambda}{1+\lambda}\left(\frac{\sin \varphi_{1} \sqrt{1+\tan ^{2} \varphi_{1}}+\mu}{\cos \varphi_{1} \sqrt{1+\tan ^{2} \varphi_{1}}-\mu \tan \varphi_{1}}\right)+\frac{1}{1+\lambda}\left(\frac{-\sin \varphi_{2} \sqrt{1+\tan ^{2} \varphi_{2}}+\mu}{\cos \varphi_{2} \sqrt{1+\tan ^{2} \varphi_{2}}+\mu \tan \varphi_{2}}\right)\right] \\
=\left[\frac{\lambda}{1+\lambda}\left(\frac{\tan \varphi_{1}+\mu}{1-\mu \tan \varphi_{1}}\right)+\frac{1}{1+\lambda}\left(\frac{-\tan \varphi_{2}+\mu}{1+\mu \tan \varphi_{2}}\right)\right]
\end{gathered}
$$

If $\varphi_{1}=\varphi_{2}$ the coefficient of friction further simplifies as:

$$
f=f_{x}=\frac{\mu}{1-(\sin \varphi)^{2}\left(1+\mu^{2}\right)}
$$

If instead $\alpha=\frac{\pi}{2}$ and $\beta=\frac{\pi}{2}$ one surface is sliding perpendicular to the other, generating forces in the $y z$-plane $\left(R_{x}=0\right)$. So the friction coefficient reduces to:

$$
f=f_{y}=\left[\frac{\lambda}{1+\lambda}\left(\frac{\mu}{\cos \varphi_{1}}\right)+\frac{1}{1+\lambda}\left(\frac{\mu}{\cos \varphi_{2}}\right)\right]
$$

and, if $\varphi_{1}=\varphi_{2}$, it becomes:

$$
f=f_{y}=\frac{\mu}{\cos \varphi}
$$

